\title{
Physical Activity, Sedentary Behavior, and Inflammatory and Hemostatic Markers in Men
}

\author{
TESSA J. PARSONS ${ }^{1,2}$, CLAUDIO SARTINI ${ }^{1,2}$, PAUL WELSH $^{3}$, NAVEED SATTAR $^{3}$, SARAH ASH $^{1}$, \\ LUCY T. LENNON ${ }^{1}$, S. GOYA WANNAMETHEE ${ }^{1}$, I-MIN LEE ${ }^{4}$, PETER H. WHINCUP ${ }^{5}$, and BARBARA J. JEFFERIS ${ }^{1,2}$ \\ ${ }^{1}$ UCL Department of Primary Care and Population Health, UCL Medical School, London, UNITED KINGDOM; ${ }^{2} U C L$ \\ Physical Activity Research Group, London, UNITED KINGDOM; ${ }^{3}$ Institute of Cardiovascular and Medical Sciences, BHF \\ Glasgow Cardiovascular Research Centre, Glasgow, UNITED KINGDOM; ${ }^{4}$ Brigham and Women's Hospital, Harvard \\ Medical School, Boston, MA; and ${ }^{5}$ Population Health Research Institute, St George's University of London, Cranmer Terrace, \\ London, UNITED KINGDOM
}

\begin{abstract}
PARSONS, T. J., C. SARTINI, P. WELSh, N. SATTAR, S. ASH, L. T. LENNON, S. G. WANNAMETHEE, I-M. LEE, P. H. WHINCUP, and B. J. JEFFERIS. Physical Activity, Sedentary Behavior, and Inflammatory and Hemostatic Markers in Men. Med. Sci. Sports Exerc., Vol. 49, No. 3, pp. 459-465, 2017. Purpose: This study aimed to determine whether higher levels of physical activity (PA) and less sedentary behavior (SB) are associated with less inflammation, indicated by inflammatory and hemostatic biomarkers, in older men. Methods: A cross-sectional study of 1139 men, from the British Regional Heart Study (mean \pm SD age $=78 \pm 5$ yr), and longitudinal analyses of 490 men with two PA measures $1 \mathrm{yr}$ apart were used in this study. Single fasting venous blood samples were analyzed for several biomarkers. PA and SB were measured using ActiGraph GT3X accelerometers. Total time and time spent in bouts of moderate to vigorous PA (MVPA), light PA, and SB were derived. Linear regression analyses were used to investigate associations. Results: Cross-sectionally, higher total PA, daily steps, and MVPA were all associated with lower levels of interleukin 6 (IL-6), C-reactive protein (CRP), tissue plasminogen activator (tPA), von Willebrand factor (vWF), and D-dimer, whereas higher levels of SB were associated with higher levels of IL-6, CRP, and tPA. Each additional 10 min of MVPA was associated with a 3.2\% lower IL-6 (95\% confidence interval $[\mathrm{CI}]=-4.5 \%$ to $-1.8 \%), 5.6 \%$ lower $\mathrm{CRP}(95 \% \mathrm{CI}=-7.8$ to -3.3$), 2.2 \%$ lower tPA $(95 \% \mathrm{CI}=-3.0$ to -1.4$), 1.2 \%$ lower vWF $(95 \% \mathrm{CI}=$ -2.1 to -0.3$)$, and $1.8 \%$ lower D-dimer $(95 \% \mathrm{CI}=-2.9$ to -0.7$)$, and for CRP, $\mathrm{vWF}$, and D-dimer independently of SB. Associations between SB and IL-6 or PPA were independent of MVPA. Longer bouts of PA or SB were not more strongly associated with outcomes than shorter bouts. Longitudinal analyses were inconsistent with these findings, possibly because of power limitations. Conclusion: Although PA (particularly MVPA) was generally associated with inflammatory and hemostatic biomarkers, we found no evidence that longer bouts were more important than shorter bouts. Key Words: EPIDEMIOLOGY, CARDIOVASCULAR DISEASE, COAGULATION, HEMOSTASIS, BIOMARKERS
\end{abstract}

$\mathrm{T}$ he aging process is associated with decreasing levels of physical activity (PA) (30) and increasing levels of inflammatory (29) and hemostatic $(22,32,33)$ markers

\footnotetext{
Address for correspondence: Tessa J Parsons, UCL Department of Primary Care and Population Health, UCL Medical School, Rowland Hill Street, London NW3 2PF, United Kingdom; E-mail: tessa.parsons@ucl.ac.uk. Submitted for publication June 2016.

Accepted for publication September 2016.

Supplemental digital content is available for this article. Direct URL citations appear in the printed text and are provided in the HTML and PDF versions of this article on the journal's Web site (www.acsm-msse.org).

0195-9131/17/4903-0459/0

MEDICINE \& SCIENCE IN SPORTS \& EXERCISE ${ }_{\circledast}$

Copyright (C) 2016 the Author(s). Published by Wolters Kluwer Health on behalf of the American College of Sports Medicine. This is an open access article distributed under the Creative Commons Attribution License 4.0 (CCBY), which permits unrestricted use, distribution, and reproduction in any medium, provided the original work is properly cited.
}

DOI: $10.1249 /$ MSS.0000000000001113 and insulin-like growth factor 1 (IGF-1) $(2,31)$, all of which have been linked to major age-related degenerative diseases such as cardiovascular disease (CVD) and type 2 diabetes. Interleukin 6 (IL-6), for example, which rises steeply with age $(12,34)$, has been shown to be particularly strongly linked to fatal CHD in older adults (29). The levels of several hemostatic factors also increase with age; tissue plasminogen activator (tPA) and D-dimer, markers of fibrinolytic activity, and von Willebrand factor (vWF), a marker of coagulation and endothelial dysfunction (34), and the balance of these factors is important because they are associated with increased risk of CHD events $(22,32,33)$. The inflammatory and coagulation systems potentially interact; increased inflammation can increase coagulation that in turn promotes inflammation, such that the systems can exert positive feedback on one another. IGF-1, a hormone stimulating cell growth and proliferation, is generally considered to have a protective effect on the cardiovascular system and declines with age (31). Several studies have reported lower IGF-1 
levels to be prospectively associated with increased CVD risk (31), although recent findings suggest this relationship may be U-shaped with both low and high levels increasing CVD risk (2).

Observational studies of how PA relates to inflammatory and hemostatic markers have been mostly based on selfreported PA data, and on middle-age rather than older adults $(14,19,23,28)$. This literature is consistent in that lower levels of PA are related to an adverse inflammatory profile and increased levels of hemostatic markers, but self-report data are limited in detecting light activities and sedentary behavior (SB), which predominate in older age $(16,18)$. Furthermore, they cannot accurately quantify the patterns of activity, e.g., whether activity is sustained in bouts of a particular duration. Intervention studies of exercise training, mostly on C-reactive protein (CRP), have shown inconsistent results and suggest that the relative contributions of PA and weight loss on CRP are unclear $(21,25)$. A review of exercise training specifically as part of cardiac rehabilitation programs and in older adults concluded that exercise training in this population was beneficial to a wide range of clinical factors including inflammation (24).

This study therefore aims to better quantify, in a sample of community-dwelling older men, associations between objectively measured PA and SB, and the inflammatory markers CRP and IL-6, hemostatic markers tPA, vWF and D-dimer, and IGF-1. We investigated associations for different intensities of PA, including moderate and vigorous activities, light activities and SB, and whether these associations were independent. We also examined the importance of bouts of activity of different durations, i.e., whether benefits are only accrued from accumulating activity in bouts of $10 \mathrm{~min}$ or more as stipulated for moderate to vigorous PA (MVPA) in the current PA guidelines (9). In addition, we examined longitudinal relationships between changes in PA and SB for $1 \mathrm{yr}$ and the previously mentioned biomarkers in a subset of men.

\section{METHODS}

Sample. The British Regional Heart Study is a populationbased cohort study following up 7735 men $(>99 \%$ white European) recruited from primary care practices in 24 British towns in 1978-1980. In 2010-2012, 3137 surviving men were invited to a physical examination, and from 2010 onward, men were asked to wear an accelerometer at yearly intervals; one of these occasions coincided with the physical examination. The National Research Ethics Service Committee London provided ethical approval. Participants provided informed written consent to the investigation in accordance with the Declaration of Helsinki.

Inflammatory and hemostatic biomarkers. Fasting venous blood samples were analyzed for IL-6 $\left(\mathrm{pg} \cdot \mathrm{mL}^{-1}\right)$, CRP $\left(\mathrm{mg} \cdot \mathrm{L}^{-1}\right)$, tPA $\left(\mathrm{ng} \cdot \mathrm{mL}^{-1}\right)$, vWF $\left(\mathrm{IU}^{\mathrm{d}} \cdot \mathrm{dL}^{-1}\right)$, D-Dimer $\left(\mathrm{ng} \cdot \mathrm{mL}^{-1}\right)$, and IGF-1 (ng. $\left.\mathrm{mL}^{-1}\right)$. CRP was assayed using ultrasensitive assay on an automated clinically validated analyzer (e411; Roche, Burgess Hill, UK) using the manufacturer's calibrators and controls (coefficient of variation 6.9\%). Plasma levels of high-sensitivity IL-6 and IGF-1 (R\&D Systems, Oxon, UK), tPA and D-dimer (Asserachrom assays; Stago, Theale, UK), and vWF antigen (Technozym assay; Pathway Diagnostics, Dorking, UK) were measured using enzyme-linked immunosorbent assays. Intra- and interassay coefficients of variation, respectively, were as follows: $5.9 \%$ and $11.6 \%$ (IL6), $5.5 \%$ and $4.1 \%$ (tPA), $14.1 \%$ and $14.3 \%$ (vWF), $5.4 \%$ and $3.2 \%$ (D-dimer), and $4.4 \%$ and $7.0 \%$ (IGF-1).

Physical activity. Men wore the GT3X accelerometer (ActiGraph, Pensacola, FL) over the right hip for $7 \mathrm{~d}$, during waking hours, removing it for swimming or bathing. Data were processed using standard methods (17). Nonwear time was excluded using the R package "physical activity" (3). Valid wear days were defined by convention as $\geq 600 \mathrm{~min}$ wear time, and participants with $\geq 3$ valid days were included in analyses. Each minute of activity was categorized using intensity threshold values of counts per minute developed for older adults: $<100$ for SB ( $<1.5$ MET), $100-1040$ for light PA (LPA) (1.5-3 MET), and >1040 for MVPA, ( $\geq 3$ MET) (6).

Other study variables. Body mass index (BMI, $\mathrm{kg} \cdot \mathrm{m}^{-2}$ ) was calculated from height (Harpenden Stadiometer) and weight in light indoor clothing (Tanita body composition analyzer (BC-418) or Tanita scales if the participant had a pacemaker or defibrillator). Participants completed a questionnaire, including information about current cigarette smoking, alcohol consumption, living alone, current use of antihypertensives, statins and anticoagulants, ever receiving a doctor diagnosis of heart attack, and heart failure or stroke (with symptoms lasting $>24 \mathrm{~h}$ ). Social class was based on longest held occupation at study entry (1978-1980), categorized as manual and nonmanual (27). Region of residence (1978-1980) was grouped into Scotland, North, Midlands, and South of England.

Statistical methods. Men reporting a diagnosis of heart attack, heart failure, or stroke (with symptoms lasting $>24 \mathrm{~h}$ ) were excluded from analyses. Descriptive statistics for demographic characteristics, inflammatory and hemostatic markers (raw values), PA, and SB were calculated by quartile of daily minutes of MVPA and SB. Associations between each of the different PA measures and each biomarker were investigated in a series of linear regression models. The PA exposures we investigated were total activity counts per day, steps per day, and minutes per day of SB, LPA and MVPA. The distributions of CRP, IL-6, tPA, vWF, and D-dimer were right skewed and therefore transformed using natural logarithm. For ease of interpretation, we estimated regression coefficients for each 10,000 counts of total activity, 1000 steps, $30 \mathrm{~min}$ of SB or LPA, and $10 \mathrm{~min}$ of MVPA and present results as percentage difference in biomarker levels derived from these regression analyses. To evaluate the independence of associations of activity intensities, models were mutually adjusted: (i) MVPA and SB and (ii) MVPA and LPA in the same model. SB and LPA were not included simultaneously because of collinearity $(r=-0.62)$. We investigated associations between biomarkers and number of minutes accumulated in bouts of MVPA, LPA, or SB for the following PA bout durations: SB lasting 1-15, 16-30, 31-60, and $\geq 61 \mathrm{~min}$; 
LPA $1-9$ and $\geq 10 \mathrm{~min}$; and MVPA $1-9$ and $\geq 10 \mathrm{~min}$. Durations of MVPA bouts were chosen to reflect current guidelines (9), but in the absence of recommendations for SB and LPA, bout durations were chosen based on their distributions. All models were adjusted for average accelerometer wear time $\left(\mathrm{min} \cdot \mathrm{d}^{-1}\right)$, season of accelerometer wear (warm, May-September, or cold, October-April), hour of blood sampling, age, region of residence, social class, living alone, smoking status, and alcohol consumption. Wear time varied markedly, and because wearing the accelerometer for longer periods allows a participant to accumulate more minutes of PA and SB, we included wear time in models to account for wear time variability. For all models including D-dimer, we excluded men taking anticoagulants (British National Formulary code 2.8.2) (1).

Finally, a subgroup of men (approximately $40 \%$ ) had accelerometer data both $1 \mathrm{yr}$ before (time 1) and at the time of (time 2) the physical examination (including blood sampling). As exploratory analyses, we investigated the relationship between change in PA/SB (time 1 to time 2) and biomarker level at time 2 (log transformed as described earlier). Linear regression models included mean activity (mean of time 1 and time 2 ) and change in activity (time 1 to time 2) and were adjusted for both mean and change in accelerometer wear time, mean age, number of days between time 1 and time 2, season (three categories: cold at both time points, warm at both time points, and different at each time point), region of residence, social class, living alone, smoking, and alcohol consumption. We present results as percentage change in biomarker per specified increase in PA or SB.

We further adjusted all models (cross-sectional and longitudinal) for BMI to investigate the effect of BMI on the relationships between PA/SB and the biomarkers. MVPA minutes were right skewed, so we repeated regression models using square root transformed MVPA.

\section{RESULTS}

We invited 3137 men to the physical examination; 1722 (55\%) attended, of whom 291 with preexisting heart disease were excluded, as were a further 157 men who either did not wear an accelerometer or did not have valid data, leaving 1274 men. We excluded 65 men who were taking anticoagulants from D-dimer analyses. Our main analyses included 1070-1139 men (depending on biomarker) with complete data for cross-sectional analysis and 455-490 men for longitudinal analysis. Of men who were invited to the examination, those with complete data had a lower BMI 10 yr earlier (26.6 vs $27.2 \mathrm{~kg} \cdot \mathrm{m}^{-2}$ ) and were more active (59\% vs $48 \%$ at least moderately active) than those who did not attend or have complete data. Men had a mean of 4938 steps and 164,749 accelerometer counts per day (Table 1) and spent on average 616, 199, and 40 min of their time in SB, LPA, and MVPA, respectively. Men who spent more time in MVPA were younger, had a lower BMI, consumed more alcohol, and were less likely to smoke or take statins, antihypertensives, or anticoagulants or have diabetes (Table 1). Relationships with SB were in the opposite direction such that men who spent more time in SB were older, had a higher BMI, and were more likely to live alone, smoke, take statins or antihypertensives, or have diabetes (see Table, Supplemental Digital Content 1, Characteristics of 1274 men without preexisting CVD or heart failure, by quartile of minutes per day spent in

TABLE 1. Characteristics of 1274 men without preexisting CVD or heart failure, by quartile of minutes per day spent in MVPA.

\begin{tabular}{|c|c|c|c|c|c|c|c|}
\hline \multirow[b]{3}{*}{ Mean (SE) or \% (n) } & \multicolumn{4}{|c|}{ Quartile of MVPA $\left(\min \cdot \mathrm{d}^{-1}\right)$} & \multirow[b]{3}{*}{$P($ Trend $)$} & \multirow[b]{3}{*}{ All Men } & \multirow[b]{3}{*}{$N$} \\
\hline & 1 & 2 & 3 & 4 & & & \\
\hline & $0.4-<3.1^{a}$ & $\geq 3.1-<30.8^{a}$ & $\geq 30.8-<53.5^{a}$ & $\geq 53.5^{a}$ & & & \\
\hline$N^{a}$ & 291 & 308 & 340 & 335 & & & 1274 \\
\hline Age, mean \pm SE $(y r)$ & $81.0 \pm 5.0$ & $78.7 \pm 4.7$ & $77.8 \pm 4.0$ & $76.5 \pm 3.5$ & $<0.0001$ & $78.4 \pm 4.6$ & 1274 \\
\hline Manual Social class, $n(\%)$ & $150(52)$ & $139(45)$ & $154(45)$ & $151(46)$ & 0.29 & $594(47)$ & 1266 \\
\hline Lives alone, $n(\%)$ & $65(23)$ & 59 (19) & $62(19)$ & $52(16)$ & 0.18 & $238(19)$ & 1256 \\
\hline Smoker, $n(\%)$ & $19(6.6)$ & $14(4.6)$ & $5(1.5)$ & $7(2.1)$ & 0.002 & $45(3.5)$ & 1257 \\
\hline Taking statins, $n(\%)$ & $156(54)$ & $136(44)$ & $150(44)$ & $129(39)$ & 0.002 & $571(45)$ & 1274 \\
\hline Taking antihypertensives, $n(\%)$ & $192(66)$ & $167(54)$ & $187(55)$ & $144(43)$ & $<0.0001$ & $690(54)$ & 1273 \\
\hline Taking anticoagulants, $n(\%)$ & $23(7.9)$ & $20(6.5)$ & $14(4.1)$ & $14(4.2)$ & 0.1 & $71(5.6)$ & 1274 \\
\hline Diabetic, $n(\%)$ & $63(22)$ & $33(11)$ & $49(14)$ & $34(10)$ & $<0.0001$ & $179(14)$ & 1273 \\
\hline Alcohol (units per week) & $5.2 \pm 7.3$ & $6.0 \pm 7.7$ & $6.8 \pm 7.5$ & $7.2 \pm 7.9$ & $<0.0001$ & $6.4 \pm 7.6$ & 1240 \\
\hline $\mathrm{BMI}\left(\mathrm{kg} \cdot \mathrm{m}^{-2}\right)$ & $23.2 \pm 4.6$ & $27.4 \pm 3.6$ & $26.9 \pm 3.6$ & $26.1 \pm 3.1$ & $<0.0001$ & $27.1 \pm 3.8$ & 1263 \\
\hline $\mathrm{IL}-6\left(\mathrm{pg} \cdot \mathrm{mL}^{-1}\right)^{b}$ & $4.30 \pm 2.15$ & $3.00 \pm 1.88$ & $2.64 \pm 1.99$ & $2.50 \pm 2.09$ & $<0.0001$ & $2.99 \pm 2.08$ & 1195 \\
\hline $\mathrm{CRP}\left(\mathrm{mg} \cdot \mathrm{L}^{-1}\right)^{b}$ & $2.16 \pm 3.40$ & $1.39 \pm 2.98$ & $1.23 \pm 3.15$ & $0.97 \pm 3.12$ & $<0.0001$ & $1.34 \pm 3.26$ & 1183 \\
\hline $\mathrm{tPA}\left(\mathrm{ng} \cdot \mathrm{mL}^{-1}\right)^{b}$ & $10.06 \pm 1.55$ & $9.33 \pm 1.52$ & $8.77 \pm 1.54$ & $8.26 \pm 1.57$ & $<0.0001$ & $9.03 \pm 1.55$ & 1190 \\
\hline $\operatorname{VWF}\left(\mathrm{IU} \cdot \mathrm{dL}^{-1}\right)^{b}$ & $129.80 \pm 1.59$ & $117.65 \pm 1.58$ & $113.94 \pm 1.61$ & $110.42 \pm 1.63$ & $<0.0001$ & $117.22 \pm 1.61$ & 1190 \\
\hline $\mathrm{D}$-dimer (ng $\left.\cdot \mathrm{mL}^{-1}\right)^{b, c}$ & $314.11 \pm 1.94$ & $228.49 \pm 1.79$ & $228.02 \pm 1.80$ & $198.83 \pm 1.69$ & $<0.0001$ & $235.59 \pm 1.84$ & 1125 \\
\hline $\mathrm{IGF}-1\left(\mathrm{ng} \cdot \mathrm{mL}^{-1}\right)$ & $72.77 \pm 24.12$ & $75.70 \pm 22.45$ & $77.11 \pm 23.88$ & $78.06 \pm 23.08$ & 0.006 & $76.08 \pm 23.63$ & 1185 \\
\hline Accelerometer wear time $\left(\min \cdot d^{-1}\right)$ & $826 \pm 71$ & $850 \pm 63$ & $863 \pm 65$ & $876 \pm 64$ & $<0.0001$ & $855 \pm 68$ & 1274 \\
\hline Total activity (counts per day) & $61,669 \pm 24,590$ & $113,645 \pm 23.416$ & $171,554 \pm 29,976$ & $294,370 \pm 83,994$ & $<0.0001$ & $164,749 \pm 99,272$ & 1274 \\
\hline Steps per day & $1895 \pm 883$ & $3645 \pm 832$ & $5302 \pm 1022$ & $8401 \pm 2370$ & $<0.0001$ & $4938 \pm 2794$ & 1274 \\
\hline SB $\left(\min \cdot d^{-1}\right)$ & $676 \pm 76$ & $638 \pm 65$ & $607 \pm 68$ & $552 \pm 76$ & $<0.0001$ & $616 \pm 84$ & 1274 \\
\hline LPA $\left(\min \cdot d^{-1}\right)$ & $144 \pm 56$ & $189 \pm 50$ & $214 \pm 52$ & $239 \pm 61$ & $<0.0001$ & $199 \pm 65$ & 1274 \\
\hline MVPA $\left(\min \cdot d^{-1}\right)$ & $7 \pm 22$ & $22 \pm 5$ & $41 \pm 6$ & $85 \pm 27$ & $<0.0001$ & $40 \pm 33$ & 1274 \\
\hline
\end{tabular}

Pearson chi-square test was used for all categorical variables except smoking for which Fisher exact test was used.

${ }^{a}$ Maximum $N$ in quartile, varies slightly with missing covariate data.

${ }^{b}$ Geometric means given.

${ }^{c}$ Men taking anticoagulants excluded. 
SB, http://links.lww.com/MSS/A774). MVPA was correlated with LPA, $r=0.50$, and LPA with SB, $r=-0.62$, both $P<0.0001$.

PA, SB, and biomarkers: cross-sectional analyses. Men who spent more time in MVPA had lower levels of IL-6, CRP, tPA, vWF, and D-dimer and higher levels of IGF-1 ( $P \leq 0.006$, Table 1). Conversely, men with higher levels of SB had higher levels of IL-6, CRP, tPA, and D-dimer and lower levels of IGF-1 $(P \leq 0.03$; see Table, Supplemental Digital Content 1, Characteristics of 1274 men without preexisting CVD or heart failure, by quartile of minutes per day spent in SB, http://links.lww.com/MSS/ A774). In regression models adjusted for covariates, higher total PA, daily steps, and MVPA were all associated with lower levels of IL-6, CRP, tPA, vWF, and D-dimer (Table 2, models 1-3). Each additional $10 \mathrm{~min}$ of MVPA was associated with a $3.2 \%$ lower IL-6, 5.6\% lower CRP, $2.2 \%$ lower tPA, 1.2\% lower vWF, and 1.8\% lower D-dimer, all $P<0.05$ (Table 2, Model 3). Each extra 30 min of LPA per day was associated with a lower IL-6, CRP, and tPA; LPA coefficients were $30 \%-50 \%$ smaller than MVPA (when MVPA coefficients were multiplied by 3 , to also relate to 30 -min increments) (Table 2, model 4). Associations between SB and IL-6, CRP, and tPA were in the opposite direction to those with LPA but of similar size (Table 2, model 5). LPA and SB were unrelated to VWF or D-dimer and none of the PA variables were associated with IGF-1.

When MVPA was included in the same model as SB (Table 2, model 6), associations between MVPA and biomarkers persisted for CRP (magnitude slightly reduced), vWF (coefficient unchanged but of borderline significance), and D-dimer (association strengthened), but not for IL-6 and tPA, whereas when MVPA was included in the same model as LPA (Table 2, model 7), all MVPA coefficients persisted, although slightly attenuated. In these latter models (Table 2, model 7), associations between LPA and IL-6 or tPA (but not for CRP) also persisted although reduced in magnitude. In models including MVPA and SB, associations between SB and IL-6 or tPA persisted, albeit with some reduction (Table 2, model 6). Additional adjustment for diabetes or use of antihypertensive medication or statins did not change results.

After adjusting for BMI, the coefficients for the associations between PA/SB and CRP or tPA were attenuated (20\%-50\% for CRP and approximately $50 \%$ for tPA), although all associations remained significant except between $\mathrm{SB}$ and $\mathrm{PAA}$ when MVPA was included (see Table, Supplemental Digital Content 2, Cross-sectional associations between physical activity intensity, sedentary time, and biomarkers, with additional adjustment for BMI, http://links.lww.com/MSS/A775). Associations between PA/SB and IL-6 were reduced to a lesser extent (15\%-30\%), whereas associations with $\mathrm{vWF}$ and D-dimer were little changed and all remained significant. Associations were essentially unchanged when models were repeated using square root transformed MVPA.

Bouts of SB and PA. MVPA lasting $\geq 10$ min was relatively uncommon; $31 \%$ accumulated $\geq 5$ bouts per week

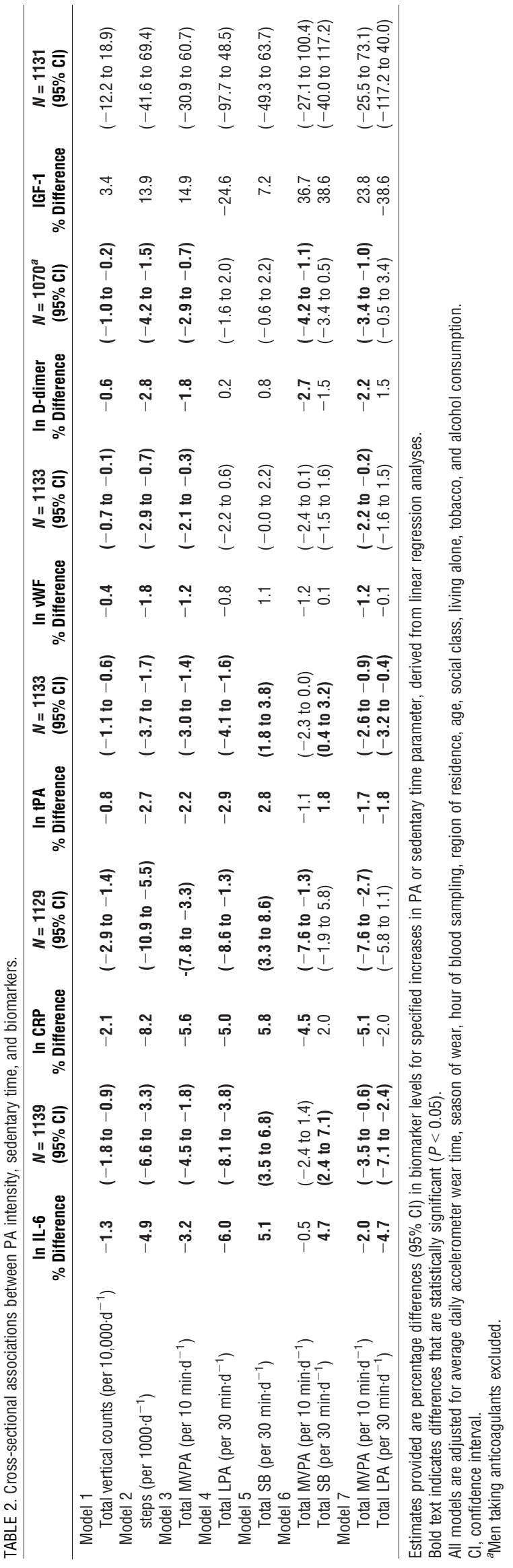


and $12 \%$ accumulated $\geq 10$ bouts per week. Regression models examining associations between number of minutes accumulated in bouts of MVPA, LPA, or SB of particular lengths and each biomarker showed no consistent evidence that accumulating activity in bouts of shorter (or longer) lengths was associated with these measures (Table 3).

Longitudinal analyses. In the subset of 455-490 men with longitudinal data, the mean time between PA measures was $327 \mathrm{~d}$ (10.8 months). The changes in percentage of time per day spent in MVPA, LPA, and SB between time 1 and time 2 were $-0.1 \%$ (SD $2.5 \%)$, LPA $-0.1 \%(4.2 \%)$, and SB $0.3 \%(5.4 \%)$, respectively (percentages account for changes in wear time). In general, associations between the biomarkers and the mean of the PA variables at time 1 and time 2 (see Table, Supplemental Digital Content 3, Associations between change in physical activity and sedentary time between time 1 and time 2, and biomarkers at time 2 in 490 men, http:// links.lww.com/MSS/A776) reflected the cross-sectional relationships (Table 2), although in the longitudinal subset the magnitude of these associations was increased for $\mathrm{PPA}, \mathrm{vWF}$, and D-dimer. Changes in activity over time were associated with vWF and IL-6; an increase in LPA of $30 \mathrm{~min}^{-1}$ between time 1 and time 2 was associated with a $3.9 \%$ decrease in vWF (see Table, Supplemental Digital Content 3, Associations between change in physical activity and sedentary time between time 1 and time 2, and biomarkers at time 2 in 490 men - model 4, http://inks.lww.com/MSS/A776), whereas an increase in $\mathrm{SB}$ was associated with a $2.8 \%$ increase in vWF and a 4.4\% decrease in IL-6 (see Table, Supplemental Digital Content 3, Associations between change in physical activity and sedentary time between time 1 and time 2, and biomarkers at time 2 in 490 men - model 5, http://links.lww. $\mathrm{com} / \mathrm{MSS} / \mathrm{A} 776)$. Adjusting for BMI reduced the magnitude and significance of the association between change in $\mathrm{SB}$ and IL-6.

\section{DISCUSSION}

In this study of older community-dwelling men, we found that higher levels of total PA, MVPA, and LPA were associated with lower levels of IL- 6 and CRP, i.e., a more favorable inflammatory profile. Conversely, higher levels of SB were associated with higher levels of these inflammatory markers. Among the hemostatic markers, this pattern of associations was also seen for $\mathrm{PPA}$, whereas for $\mathrm{vWF}$ and D-dimer, only total PA and MVPA (not LPA or SB) were associated with marker levels. We found some evidence that associations between biomarkers and PA were independent of SB and vice versa, although this was not entirely consistent across inflammatory or hemostatic markers; associations between MVPA and CRP, vWF, or D-dimer were independent of SB or LPA (borderline significance in the case of MVPA and vWF adjusted for SB), whereas for IL-6 and tPA, the association with SB was independent of MVPA, and associations with MVPA and LPA were independent of each other. Our findings are consistent with previous studies; higher levels of

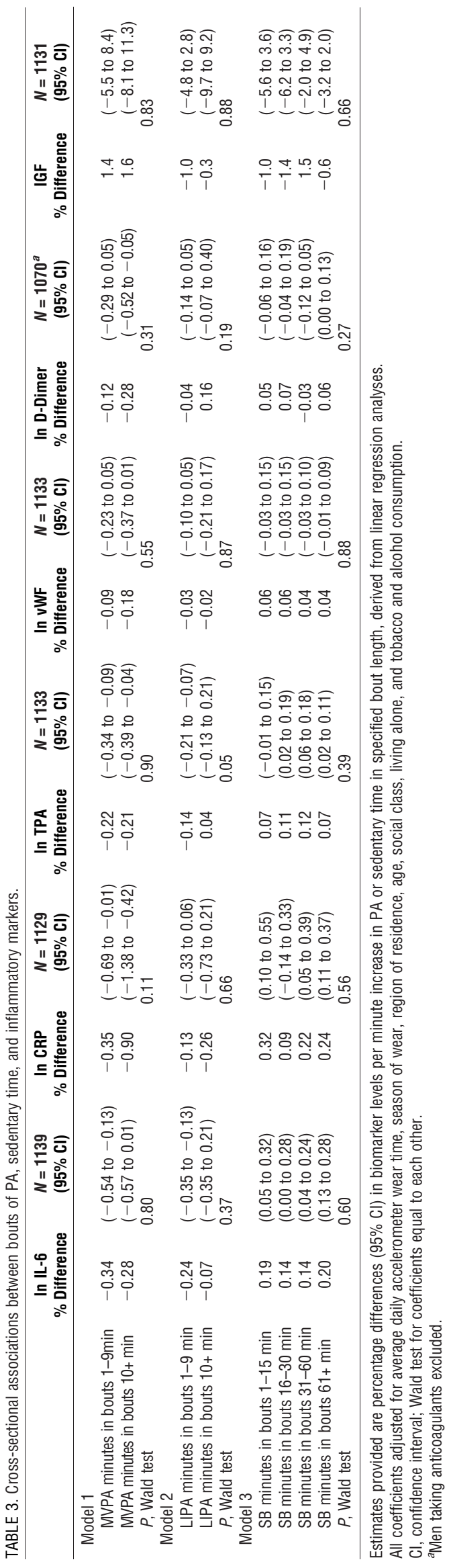


PA were associated with lower levels of inflammatory and hemostatic markers $(5,11,19,23,26)$. In our study, BMI reduced magnitude of associations between PA/SB and CRP, tPA, and IL-6, not unexpectedly because BMI is directly related to inflammatory marker levels and adipose tissue is a source of IL-6 production. Although some studies report that BMI attenuates associations $(5,11)$, not all do (26). We found no evidence that accumulating PA in longer bouts was of greater benefit than shorter bouts, but we acknowledge that our statistical power may be limited in this respect.

Strengths and limitations. The major strengths of our study are (i) the presentation of relationships between objectively measured PA of different intensities, SB, and a range of inflammatory and hemostatic biomarkers and (ii) the examination of the importance of activity accumulated in bouts of different lengths relating to current PA guidelines. The men in our study are from a less-studied older age-group and a population sample that increases generalizability, although possibly not to younger ages or women. Although men who participated in our study were healthier than those who did not, given the wide range of activity levels among participants, we would not anticipate any bias in average level of activity to affect our estimation of associations between activity and biomarkers. As is typical in older populations, amounts of moderate and vigorous activity were small, and we therefore present them combined, which also allows comparison with other studies. The ActiGraph accelerometer is validated for measuring low levels of energy expenditure but lacks good inclinometer data to differentiate standing and sitting during periods of $<100$ counts per minute. However, the mean value of these minutes was $<10$ counts per minute, suggesting that SB time was very sedentary. Our response rate for agreeing to wear an accelerometer was 51\% (17), greater than rates reported in other UK studies of older adults $(7,8)$, and adherence to the $7 \mathrm{~d}$ accelerometer wear protocol was high, with $96 \%$ of men providing the $\geq 5 \mathrm{~d}$ of data needed to predict habitual PA/SB (15). We were able to adjust for a range of potential confounding factors, although we are unable to establish the direction of effect from our cross-sectional analyses. We explored direction of effect in longitudinal analyses, but our findings for associations between $1 \mathrm{yr}$ change in PA/ SB and marker levels were not consistent with our crosssectional findings, possibly partly because of the small changes in PA and SB. Given the small sample size and limited followup period, we consider these to be secondary analyses which require further investigation in larger studies.

Implications of findings. Physical activity may exert its protective effect on CVD in part through antiinflammatory effects and by influencing hemostatic factors. Inflammation has been linked to CVD, insulin resistance, chronic obstructive pulmonary disease, some cancers, dementia, and depression, and PA has been associated with a decreased risk of these conditions (13). The activation of the coagulation system and the resulting increase in hemostatic markers have been implicated in the development of atherosclerosis, and higher levels of markers such as D-dimer have been associated with greater declines in physical function (4). The inflammatory and coagulation pathways become more active with aging and potentially exert positive feedback on each other. For example, D-dimer stimulates the synthesis and release of proinflammatory cytokines, including IL-6, which promotes CRP, coagulation factor, and platelet synthesis and contributes to endothelial dysfunction $(10,20)$. PA reduces CRP levels via multiple possible mechanisms, including a decrease in cytokine production by adipose tissue and skeletal muscle, improved endothelial function and insulin sensitivity, and an antioxidant effect.

Our study shows PA, in particular MVPA, to be associated with more favorable profiles of inflammatory and hemostatic markers, which identify risk for CVD and other chronic age-related diseases. In our data, longer bouts of PA or SB were not more strongly associated with biomarkers than shorter bouts. In longitudinal analyses, increases in PA were beneficial only for vWF, and interestingly, it was LPA and SB showing this association (an increase SB being detrimental), suggesting they too may have relevance, although further studies are needed. Given the decline in PA in aging populations, PA is an important potential modifier of adverse inflammatory and hemostatic risk profiles.

\section{CONCLUSION}

In community-dwelling older British men, objectively measured PA showed beneficial associations and SB detrimental associations with inflammatory and hemostatic biomarkers, including IL-6, CRP, tPA, vWF, and D-dimer. Adverse profiles of these markers have been linked to several major age-related degenerative diseases ranging from CVD to dementia. This is the first study to investigate associations between a range of biomarkers and objectively measured PA, including different intensities (MVPA, LPA, and SB) and number of minutes per day accumulated in bouts of specific durations, about national PA guidelines. We found no evidence that the bout length of PA or SB was important for the biomarkers we examined. Our findings indicate that all activity matters, particularly MVPA. Current guidelines recommend that MVPA is accumulated in bouts of at least $10 \mathrm{~min}$; therefore, our findings are relevant for considerations of future guideline refinements. PA may be an important potential modifier of adverse inflammatory and hemostatic risk profiles.

The authors acknowledge the British Regional Heart Study team for data collection. This work was supported by the British Heart Foundation (grant nos. PG/13/86/30546, PG09/024, and RG/08/ 013/25942) and the National Institutes of Health Research (postdoctoral fellowship no. 2010-03-023). The funders had no role in the design, methods, subject recruitment, data collections, analysis, or preparation of this article. The views expressed in this publication are those of the authors and not necessarily those of the funders.

The authors declare there is no conflict of interest and that the results of the study are presented clearly, honestly, and without fabrication, falsification, or inappropriate data manipulation. The results of the study do not constitute endorsement by the American College of Sports Medicine. 


\section{REFERENCES}

1. British National Formulary (BNF) [Internet]. 2016 [cited 2016 May 27]. Available from https://www.evidence.nhs.uk/formulary/ bnf/current/Cited.

2. Carlzon D, Svensson J, Petzold M, et al. Both low and high serum IGF-1 levels associate with increased risk of cardiovascular events in elderly men. $J$ Clin Endocrinol Metab. 2014;99(11):E2308-16.

3. Choi L, Liu Z, Matthews CE, Buchowski MS. Physical Activity: Process Physical Activity Accelerometer Data (0.1-1) [Internet]. 2011 [2016 September 19]. Available from http://cran.r-project.org/ accessed.

4. Cohen HJ, Harris T, Pieper CF. Coagulation and activation of inflammatory pathways in the development of functional decline and mortality in the elderly. Am J Med. 2003;114(3):180-7.

5. Colbert LH, Visser M, Simonsick EM, et al. Physical activity, exercise, and inflammatory markers in older adults: findings from the Health, Aging and Body Composition Study. J Am Geriatr Soc. 2004;52(7):1098-104.

6. Copeland JL, Esliger DW. Accelerometer assessment of physical activity in active, healthy older adults. J Aging Phys Act. 2009; 17(1):17-30.

7. Craig R, Mindell J, Hirani V. Health Survey for England 2008. Physical Activity and Fitness. Summary of Key Findings London [Internet]. 2009 [cited 2016 May 27]. Available from: http:// wwwhscicgovuk/catalogue/PUB00430/heal-surv-phys-acti-fitneng-2008-rep-v1pdf.

8. Davis MG, Fox KR, Hillsdon M, Sharp DJ, Coulson JC, Thompson JL. Objectively measured physical activity in a diverse sample of older urban UK adults. Med Sci Sports Exerc. 2011;43(4):647-54.

9. Department of Health. Start Active, Stay Active: A Report on Physical Activity from the Four Home Countries' Chief Medical Officers. London, UK: Department of Health; 2011 [cited 2016 May 27]. Available from https://www.gov.uk/government/uploads/system/ uploads/attachment_data/file/216370/dh_128210.pdf.

10. Eder K, Baffy N, Falus A, Fulop AK. The major inflammatory mediator interleukin-6 and obesity. Inflamm Res. 2009;58(11): 727-36.

11. Eliasson M, Asplund K, Evrin PE, Lindahl B, Lundblad D. Hyperinsulinemia predicts low tissue plasminogen activator activity in a healthy population: the Northern Sweden MONICA Study. Metabolism. 1994;43(12):1579-86.

12. Ferrucci L, Corsi A, Lauretani F, et al. The origins of age-related proinflammatory state. Blood. 2005;105(6):2294-9.

13. Gleeson M, Bishop NC, Stensel DJ, Lindley MR, Mastana SS, Nimmo MA. The anti-inflammatory effects of exercise: mechanisms and implications for the prevention and treatment of disease. Nat Rev Immunol. 2011;11(9):607-15.

14. Hamer M. The relative influences of fitness and fatness on inflammatory factors. Prev Med. 2007;44(1):3-11.

15. Hart TL, Swartz AM, Cashin SE, Strath SJ. How many days of monitoring predict physical activity and sedentary behavior in older adults? Int J Behav Nutr Phys Act. 2011;8:62.

16. Harvey JA, Chastin SF, Skelton DA. How sedentary are older people? A systematic review of the amount of sedentary behavior. J Aging Phys Act. 2015;23(3):471-87.

17. Jefferis BJ, Sartini C, Lee IM, et al. Adherence to physical activity guidelines in older adults, using objectively measured physical activity in a population-based study. BMC Public Health. 2014;14:382.
18. Jefferis BJ, Sartini C, Shiroma E, Whincup PH, Wannamethee SG, Lee IM. Duration and breaks in sedentary behaviour: accelerometer data from 1566 community-dwelling older men (British Regional Heart Study). Br J Sports Med. 2015;49:1591-4.

19. Jefferis BJ, Whincup PH, Lennon LT, Papacosta O, Goya Wannamethee S. Physical activity in older men: longitudinal associations with inflammatory and hemostatic biomarkers, $\mathrm{N}$-terminal pro-brain natriuretic peptide, and onset of coronary heart disease and mortality. J Am Geriatr Soc. 2014;62(4):599-606.

20. Kanapuru B, Ershler WB. Inflammation, coagulation, and the pathway to frailty. Am J Med. 2009;122(7):605-13.

21. Lavie CJ, Church TS, Milani RV, Earnest CP. Impact of physical activity, cardiorespiratory fitness, and exercise training on markers of inflammation. J Cardiopulm Rehabil Prev. 2011;31(3):137-45.

22. Lowe GD, Yarnell JW, Sweetnam PM, Rumley A, Thomas HF, Elwood PC. Fibrin D-dimer, tissue plasminogen activator, plasminogen activator inhibitor, and the risk of major ischaemic heart disease in the Caerphilly Study. Thromb Haemost. 1998;79(1): 129-33.

23. May M, Lawlor DA, Patel R, Rumley A, Lowe G, Ebrahim S. Associations of von Willebrand factor, fibrin D-dimer and tissue plasminogen activator with incident coronary heart disease: British Women's Heart and Health cohort study. Eur J Cardiovasc Prev Rehabil. 2007;14(5):638-45.

24. Menezes AR, Lavie CJ, Forman DE, Arena R, Milani RV, Franklin BA. Cardiac rehabilitation in the elderly. Prog Cardiovasc Dis. 2014;57(2):152-9.

25. Milani RV, Lavie CJ, Mehra MR. Reduction in C-reactive protein through cardiac rehabilitation and exercise training. $J$ Am Coll Cardiol. 2004;43(6):1056-61.

26. Mora S, Lee IM, Buring JE, Ridker PM. Association of physical activity and body mass index with novel and traditional cardiovascular biomarkers in women. JAMA. 2006;295(12):1412-9.

27. Office of Population Censuses and Surveys. Classification of Occupations 1970. Great Britain (London): HMSO; 1971.

28. Plaisance EP, Grandjean PW. Physical activity and high-sensitivity C-reactive protein. Sports Med. 2006;36(5):443-58.

29. Sattar N, Murray HM, Welsh P, et al. Are markers of inflammation more strongly associated with risk for fatal than for nonfatal vascular events? PLoS Med. 2009;6(6):e1000099.

30. Shiroma EJ, Lee IM. Physical activity and cardiovascular health: lessons learned from epidemiological studies across age, gender, and race/ethnicity. Circulation. 2010;122(7):743-52.

31. Ungvari Z, Csiszar A. The emerging role of IGF-1 deficiency in cardiovascular aging: recent advances. J Gerontol A Biol Sci Med Sci. 2012;67(6):599-610.

32. Wannamethee SG, Whincup PH, Lennon L, Rumley A, Lowe GD. Fibrin D-dimer, tissue-type plasminogen activator, von Willebrand factor, and risk of incident stroke in older men. Stroke. 2012;43(5): 1206-11.

33. Whincup PH, Danesh J, Walker M, et al. von Willebrand factor and coronary heart disease: prospective study and meta-analysis. Eur Heart J. 2002;23(22):1764-70.

34. Yarnell JW, Sweetnam PM, Rumley A, Lowe GD. Lifestyle and hemostatic risk factors for ischemic heart disease: the Caerphilly Study. Arterioscler Thromb Vasc Biol. 2000;20(1):271-9. 